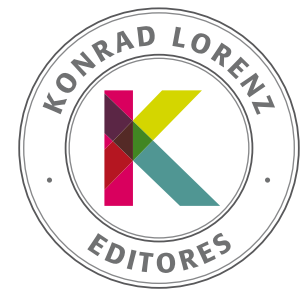

\title{
Evaluación de las propiedades psicométricas de la Escala de Abuso Online en Relaciones de Noviazgo en adolescentes y adultos jóvenes colombianos
}

\author{
César Armando Rey Anacona*, Jorge Arturo Martínez Gómez, Eliana Marcela Ocampo Rojas
}

Universidad Pedagógica y Tecnológica de Colombia, Tunja, Colombia

Recibido el 1 de octubre de 2020; aceptado el 26 de abril de 2021

PALABRAS CLAVE:

Violencia en el noviazgo, adolescentes, adultos jóvenes, propiedades psicométricas, ciberviolencia

\section{KEYWORDS:}

Dating violence, adolescents, young adults, psychometric properties, cyberviolence
Resumen Introducción/objetivo: Los estudios realizados en algunos países, incluyendo a Colombia, indican altas prevalencias de ciberviolencia en el noviazgo. Sin embargo, en dicho país no se cuenta con instrumentos validados para medir esta forma de violencia. El objetivo de este estudio fue evaluar la confiabilidad y la validez de la Escala de Abuso Online en Relaciones de Noviazgo. Método: Participaron 2023 estudiantes de colegio y universitarios (53\% mujeres), entre 13 y 40 años. Resultados: El análisis factorial exploratorio demostró la conveniencia de conservar la estructura original de 20 ítems de las dos escalas del instrumento (perpetración y victimización) y las dos subescalas de cada una ("agresiones directas" y "monitoreo/control”), estructura que fue ratificada por el análisis factorial confirmatorio con índices adecuados de ajuste. Además, las cuatro subescalas correlacionaron significativamente entre sí y con las escalas de la Lista de Chequeo de Experiencias de Maltrato Psicológico en la Pareja. Los índices alfa oscilaron entre .75 y .87. Conclusiones: Estos resultados indican que el instrumento presenta unas propiedades psicométricas que avalan su uso con adolescentes y adultos jóvenes colombianos.

(C) 2021 Fundación Universitaria Konrad Lorenz. Este es un artículo Open Access bajo la licencia CC BY-NC-ND (http://creativecommons.org/licenses/by-nc-nd/4.0/).

Evaluation of the psychometric properties of the Cyber Dating Abuse Questionnaire in Colombian adolescents and young adults

Abstract Introduction/objective: Studies carried out in some countries, including Colombia, indicate high prevalences of cyber dating abuse. However, in that country there are no validated instruments to measure this form of violence. The objective of this study was to evaluate the reliability and validity of the Cyber Dating Abuse Questionnaire. Method: The participants

* Autor para correspondencia.

Correo electrónico: cesar.rey@uptc.edu.co 
were 2023 college and university students (53\% female), 13-40 years aged. Results: The exploratory factor analysis showed the convenience of preserving the original structure of 20 items on the two scales of the instrument (perpetration and victimization) and the two subscales of each ("direct aggression" and "monitoring/control"). This structure was ratified by confirmatory factor analysis with adequate adjustment indices. In addition, the four subscales correlated significantly with each other and with the scales of the Checklist of Experiences of Psychological Abuse in the Couple. Alpha indices ranged from .75 to .87. Conclusions: These results indicate that the instrument has psychometric properties that ensure its use with Colombian adolescents and young adults.

(C) 2021 Fundación Universitaria Konrad Lorenz. This is an open access article under the CC BY-NC-ND license (http://creativecommons.org/licenses/by-nc-nd/4.0/).

En el siglo XXI, las tecnologías de la información y las comunicaciones (TIC) como la Internet, las aplicaciones de mensajería inmediata y las redes sociales se han convertido en uno de los principales medios para socializar de los adolescentes y adultos jóvenes. Lo anterior ha permitido no solo interactuar rápidamente con familiares, amigos y una gran diversidad de personas, sino también establecer relaciones de amistad y de pareja, y compartir información de diversa índole con diferentes propósitos (Peña, et al, 2018; Reed, et al., 2017; Stonard et al., 2014). La facilidad en las interacciones por estos medios también ha favorecido las comunicaciones en las relaciones de pareja, de manera que cada integrante puede conocer más fácilmente las relaciones interpersonales y las actividades del otro(a). No obstante, estos mismos medios también se han utilizado con propósitos oscuros, situación que ha generado diferentes formas de violencia por medios electrónicos o ciberviolencia, tales como el cyberbullying, el sexting y el grooming (Borrajo \& Gámez-Gaudix, 2016; Luengo, 2014; Peña et al., 2018; Stonard et al., 2014).

Entre tanto, la violencia en el noviazgo (VN) comprende todos aquellos actos de violencia física, psicológica o sexual que ocurren en una pareja que tiene una relación íntima o que se cita para salir. Dentro de estas conductas, se incluye el acecho a la pareja (Centers for Disease Control and Prevention, 2019; Gracia-Leiva et al., 2019). Esta forma de violencia se ha constituido como un tema de primordial interés para muchos investigadores, debido a las elevadas cifras de prevalencia y las dificultades de salud asociadas a este fenómeno (Hoefer et al., 2015; Rubio-Garay et al., 2017; Wincentak et al., 2017).

La VN no solamente puede presentarse en persona sino también electrónicamente, por medio de comportamientos realizados con el fin de intimidar, agredir o controlar a la pareja mediante el uso de dispositivos electrónicos como teléfonos celulares, tabletas y computadores conectados a Internet (Centers for Disease Control and Prevention, 2019; Borrajo, Gámez-Guadix \& Calvete, 2015), lo que da lugar a la ciberviolencia en el noviazgo (Borrajo, Gámez-Guadix, Pereda et al., 2015), abuso online en el noviazgo (Borrajo \& Gámez-Gaudix, 2015), digital dating abuse (Reed et al., 2017) o technology assisted adolescent dating violence and abuse (Stonard et al., 2014). Borrajo, Gámez-Guadix, Pereda et al. (2015), las conductas de ciberviolencia en el noviazgo (CVN), se pueden dividir en (a) las agresiones directas, como los insultos enviados a través de mensajes de texto, o la difusión de información negativa sobre la pareja, y (b) las conductas de monitoreo y control sobre esta, como revisar su muro en las redes sociales y rastrear su ubicación con alguna aplicación de teléfonos celulares.

Debido a que estas conductas son manifestaciones de violencia psicológica, se ha considerado que la CVN es una extensión de la VN offline de tipo psicológico (Temple et al., 2016). En concordancia con lo anterior, Caridade et al. (2020) encontraron una ocurrencia alta de estos dos tipos de violencia entre 145 adolescentes y adultos jóvenes portugueses; mientras que Borrajo, Gámez-Guadix, Pereda et al. (2015) hallaron correlaciones estadísticamente significativas entre las dos, entre 788 españoles de 18 a 30 años.

Los resultados de algunos estudios señalan diferencias en las variables asociadas y las manifestaciones de la VN entre adolescentes y adultos jóvenes que se podrían explicar por diferencias madurativas y el número de experiencias románticas (Redondo et al., 2017a; Villa et al., 2017). Así, se encontraron mayores tasas de dependencia emocional y VN entre estudiantes de secundaria comparados con universitarios (Villa et al., 2017), mientras que Dardis et al. (2017) hallaron que las experiencias de victimización previas influían en la percepción negativa de los actos de VN entre universitarios. Por lo tanto, es previsible que existan diferencias entre adolescentes y adultos jóvenes en la CVN, las cuales se deberían examinar.

Stonard et al. (2014) llevaron a cabo una revisión sobre la naturaleza, la prevalencia y el impacto de la CVN en la adolescencia, por lo mismo, reseñaron 13 estudios en los que se midió este tipo de violencia, la mayoría con medidas ad hoc, realizados en Estados Unidos y el Reino Unido. Estos estudios reportaron tasas de victimización que oscilaban entre el $12 \%$ y el $56 \%$ y de perpetración de entre el $12 \%$ y el $54 \%$. Allí también se encontró que eran más altas las tasas de mujeres víctimas de esta forma de violencia, particularmente la de tipo sexual, la cual fue ejercida por los hombres en un porcentaje mayor. Caridade et al. (2019), por su parte, efectuaron una revisión con 44 estudios publicados sobre el tema, entre los cuales se informaron cifras de victimización que oscilaban entre el $5.8 \%$ y el $92 \%$ y de perpetración de entre el $8.1 \%$ y el $93.7 \%$, en algunos estudios no se encontraron diferencias por sexo en dichas tasas.

Aunque faltan más investigaciones sobre el tema, es previsible que las ciberagresiones y el cibercontrol generen reacciones emocionales negativas (Hinduja \& Patchin, 2020; Stonard et al., 2014). Borrajo y Gámez-Gaudix (2016) encontraron entre 782 españoles de 18 a 30 años que la CVN se relacionaba con mayores niveles de depresión y ansiedad y un peor ajuste diádico, mientras que Smith et al. (2018) hallaron, entre 190 adolescentes canadienses de 14 
a 18 años, que la victimización se asociaba con una baja autoestima y malestar psicológico. Reed et al. (2017), por su parte, encontraron entre 703 estudiantes estadounidenses de 13 a 19 años que la CVN generaba más indisposición y reacciones emocionales negativas entre las mujeres que entre los hombres.

A pesar de la evidencia que indica que la CVN es muy frecuente entre los adolescentes y adultos jóvenes y su posible impacto negativo, hay pocos instrumentos validados dirigidos a obtener información sobre este tipo de violencia (Cava \& Buelga, 2018). Gámez-Gaudix et al. (2018) realizaron una revisión de estudios sobre el abuso online en la pareja y encontraron varios instrumentos que medían la victimización o la perpetración, pero la mayoría se centraba en aspectos específicos de este tipo de violencia, como las conductas de control ejercidos a través de Facebook y algunos no reportaban índices de validez, solo de confiabilidad. Dentro de estos instrumentos, 13 medían tanto la victimización como la perpetración, de los cuales cuatro se desarrollaron en España y México. Dos de ellos informaron índices de confiabilidad y validez, incluyendo la Escala de Abuso Online en Relaciones de Noviazgo de Borrajo, Gámez-Guadix, Pereda et al. (2015). Una revisión posterior efectuada por Caridade et al. (2019), con 44 estudios sobre CVN, analizó un total de 17 instrumentos, de los cuales nueve medían tanto la perpetración como la victimización y entre los cuales se encontraban los cuatro instrumentos desarrollados en España y México; allí la escala mencionada es el instrumento más utilizado, pues fue incluido en nueve estudios. Esta misma escala fue validada más recientemente con 1538 estudiantes de secundaria y universitarios chilenos (Lara, 2020).

En Colombia no se cuenta con instrumentos validados para examinar la CVN, a pesar de que los estudios de prevalencia realizados indican que la VN podría afectar a un importante número de adolescentes y adultos jóvenes (e.g., Martínez et al., 2016; Rey, 2009). Por ejemplo, un estudio realizado con 237 estudiantes universitarios entre 16 y 28 años, residentes de la ciudad de Bucaramanga (Redondo et al., 2017b), encontró que el $94.9 \%$ de los participantes había ejercido violencia verbal, mientras que el $34.7 \%$ había ejecutado actos de violencia relacional y el $22 \%$ conductas de violencia física. También hallaron que el $91.9 \%$ de los participantes había sido víctima de violencia verbal, el $45.3 \%$ de violencia relacional y el $17.8 \%$ de violencia física.

La única investigación publicada sobre la prevalencia de la CVN en Colombia, en el que participaron 639 estudiantes universitarios, con un promedio de 17.66 años (Redondo et al., 2017c), encontró que el $27.5 \%$ de los participantes habían sido agredidos en alguna ocasión en el último año y que el $26.7 \%$ había ejercido este tipo de violencia en el mismo período de tiempo, aunque la victimización solía ser inferior a un mes y de intensidad moderada. Sin embargo, los instrumentos utilizados en este estudio no han sido validados con muestras colombianas.

Un estudio de la Asociación de la Industria Móvil de Colombia (2017) reveló que el celular es el principal medio de información para el $66 \%$ de la población colombiana y que, en promedio, los colombianos utilizan el celular 4.3 horas al día; específicamente 4.97 horas las personas entre 18 y 35 años. Otra encuesta realizada por la empresa Deloitte (2019), entre 928 usuarios de telefonía móvil de 16 a
45 años, encontró que el $57 \%$ consultaba las redes sociales y que el $76 \%$ consideraba que usaban demasiado sus teléfonos celulares, datos que indican que la población adolescente y adulta joven en Colombia podría estar expuesta a diferentes formas de ciberviolencia, particularmente la CVN.

El Cyber Dating Abuse Questionnaire o Escala de Abuso Online en Relaciones de Noviazgo de Borrajo, Gámez-Guadix, Pereda et al. (2015) es un cuestionario de autoinforme en castellano que examina tanto las agresiones directas como las conductas de control por medios electrónicos. Además, examina tanto la victimización como la perpetración, un aspecto clave, ya que en un importante número de casos la VN es bidireccional (Alegría \& Rodríguez, 2015; Rubio-Garay et al., 2017), así como los posibles motivos que conllevaron a dicha perpetración o victimización. Sus autoras informaron índices adecuados de confiabilidad y validez con una muestra de 788 españoles de 18 a 30 años. No obstante, este instrumento no ha sido validado con muestras colombianas y sus autoras no examinaron sus cualidades psicométricas entre personas menores a 18 años, a pesar de que la CVN es muy frecuente entre los adolescentes, como lo señalan los resultados de la revisión de Stonard et al. (2014), ni entre los adultos de 30 a 40 años, aunque el uso de las TIC también está muy extendido entre los adultos jóvenes. De hecho, algunos estudios señalan altas prevalencias de VN en estas edades en Colombia (Rey-Anacona, 2013; Martínez-Gómez et al., 2021).

Conforme a lo anterior, este estudio se planteó como objetivo evaluar la validez y la confiabilidad de la Escala de Abuso Online en Relaciones de Noviazgo (Borrajo, Gámez-Guadix, Pereda et al. 2015), con una muestra de adolescentes y adultos jóvenes colombianos entre 13 y 40 años.

\section{Método}

\section{Diseño}

De acuerdo con la tipología de estudios en psicología planteada por Montero y León (2007), se llevó a cabo un estudio instrumental dirigido a examinar las propiedades psicométricas de la escala mencionada.

\section{Participantes}

Participaron un total de 2023 adolescentes y adultos jóvenes entre 13 y 40 años, de los cuales 1000 vivían en Bogotá (capital de Colombia) y 1023 en la ciudad de Tunja (departamento de Boyacá). De estos, 1072 eran mujeres (53\%) y 951 hombres (47\%); 1511 (74.7\%) eran adolescentes (entre 13 y 19 años) y 512 (25.3\%) adultos jóvenes (entre 20 y 40 años), con un promedio de edades de 17.92 años ( $S D=3.6$ años). El $61 \%(n=1234)$ eran estudiantes de seis instituciones de educación media de Bogotá y de Tunja, que cursaban entre octavo y undécimo grado, mientras que el $39 \%(n=789)$ eran estudiantes de diferentes licenciaturas y carreras universitarias de Tunja. Todos los participantes reportaron que llevaban un promedio de 11.65 meses con su pareja actual y 9.48 meses con su pareja anterior, e informaron haber tenido un promedio de 4.17 relaciones de pareja. En su mayoría, declararon una orientación heterosexual $(n=1868,92.3 \%)$, 
mientras que el $2 \%(n=40)$ se consideró homosexual y un $5.4 \%$ bisexual ( $n=109$; seis participantes no informaron su orientación sexual).

Los participantes vivían en sectores de nivel socioeconómico bajo-bajo ( $n=416,20.6 \%$ ), bajo ( $n=1011,50 \%$, medio-bajo ( $n=472,23.3 \%)$, medio $(n=100,4.9 \%)$ y medio-alto ( $n=16,0.8 \%$ ), según la clasificación del Departamento Administrativo Nacional de Estadística (ocho participantes no informaron el nivel). Los criterios de inclusión y exclusión fueron los siguientes: (a) tener entre 13 y 40 años, (b) ser solteros, (c) haber tenido o tener una relación amorosa y (d) contar con el consentimiento escrito de los padres y el asentimiento del(a) adolescente (excepto los mayores de edad). La selección de estos participantes se realizó a partir de un muestreo no aleatorio, según su disponibilidad en las instituciones participantes y no se les recompensó económicamente por su participación.

\section{Instrumentos}

Cyber Dating Abuse Questionnaire - CDAQ (Escala de Abuso Online en Relaciones de Noviazgo; Borrajo, GámezGuadix, Pereda et al., 2015) es un cuestionario que recoge información sobre la victimización y la perpetración de CVN, en el último año de la relación, a través de dos subescalas conformadas por diez ítems cada una: (a) las agresiones directas como insultos y amenazas y (b) las conductas de monitoreo/control como mirar la hora en la que la pareja se desconectó de una red social, entre otras. La escala contiene 20 parejas de ítems paralelos, para reportar la victimización o la ejecución de cada una de las formas de maltrato contempladas en el instrumento, los cuales se contestan con una escala tipo Likert con seis opciones de respuesta: "nunca" = 1, "no en el último año, pero sí anteriormente" = 2, "raramente: 1 о 2 veces" = 3, "algunas veces: entre 3 a 10 veces" = 4, "usualmente: entre 10 a 20 veces" = 5 y “siempre: más de 20 veces" = 6 . También permite informar los motivos de las agresiones recibidas y perpetradas, incluyendo celos, sentimientos como enfado y frustración, retaliación por una agresión similar, juego o broma, la personalidad y otros. Sus autoras reportaron que las subescalas del instrumento correlacionaron significativamente con escalas de VN psicológica offline y cyberbullying, conforme con los datos obtenidos con la muestra ya señalada y evidenciando índices alfa que oscilaban entre .73 y .87.

Checklist of Experiences of Psychological Abuse to the Couple - CEPA (Lista de Chequeo de Experiencias de Maltrato Psicológico en la Pareja; Rey et al., 2019) permite reportar la realización de 14 conductas de maltrato psicológico, agrupadas en tres subescalas obtenidas a través del análisis factorial confirmatorio, con adecuados índices de ajuste: (a) Coerción (4 ítems), (b) Humillación (5 ítems) y (c) Control ( 5 ítems). Los ítems se responden con una escala Likert con cuatro opciones de respuesta: "Nunca" = 0, "Una vez" = 1, "Algunas veces" = 2 y "Muchas veces" = 3. Rey et al. (2019) informaron alfas que oscilaban entre .71 y .83., con una muestra de 1729 hombres y mujeres colombianos, entre 12 y 42 años. Las subescalas correlacionaron significativamente entre sí y con escalas de rasgos de personalidad asociados al género, lo que aportó evidencias a favor de su validez de constructo. En esta investigación se utilizó esta lista de chequeo para examinar la validez de constructo de la CDAQ (Borrajo, Gámez-Guadix, Pereda et al., 2015), ya que la CVN se ha considerado una extensión de la VN offline, particularmente la de tipo psicológico (Caridade et al., 2020; Temple et al., 2016).

\section{Procedimiento}

Inicialmente, se revisó el castellano del instrumento y se efectuaron las siguientes adaptaciones lingüísticas: (a) cambio de las palabras "hayáis" y "habéis" de las instrucciones por "que hayan" y "han", respectivamente: (b) reemplazo de las palabras "actualizaciones de estado" del ítem 1 por "publicaciones", para obtener: "Mi pareja o expareja ha controlado las publicaciones del muro de mi red social" y "He controlado las publicaciones del muro de la red social de mi pareja o expareja"; (c) cambio de la palabra "comprometidas" por "comprometedoras" en el ítem 6; (d) reemplazo de la palabra "comprometida" por "comprometedora" en el ítem 8 y (e) cambio de la palabra "colgado" por “publicado" en el ítem 16.

Posteriormente, se solicitó la autorización para la realización del estudio en varias instituciones de educación media y universitaria de Bogotá y Tunja, tras lo cual se contactó a los estudiantes en sus salones de clase, para presentarles la investigación y entregarles un formato de consentimiento informado que tenía que ser firmado por los padres, en el caso de los menores de edad. Este formato informaba sobre los objetivos y la metodología del estudio, la confidencialidad de los datos obtenidos, la independencia de la investigación con respecto a la institución, la voluntariedad de la participación y el respeto a la decisión de retirarse en el transcurso de esta sin ninguna consecuencia legal o social. También se les garantizó que sus datos de identificación no aparecerían en ningún informe, presentación o publicación académica del estudio y que la información recogida iba a ser utilizada solo con fines investigativos y de formación académica, en cumplimiento de las normas legales que rigen la investigación científica en Colombia. Luego se procedió a aplicar los instrumentos de manera grupal y anónima, revisando que los participantes contestaran todos los ítems y ofreciéndoles la posibilidad de recibir los resultados de la aplicación de los instrumentos, si lo deseaban.

\section{Análisis de datos}

Los datos obtenidos se incorporaron en una base de datos de SPSS versión 22.0. Estos se revisaron para identificar errores de captura de información y se hicieron las correcciones correspondientes teniendo en cuenta las respuestas dadas a los instrumentos aplicados. Para evaluar la validez de constructo, se implementaron inicialmente las pruebas de adecuación de Kaiser-Meyer-Olkin (KMO) y la prueba de esfericidad de Bartlett con el fin de examinar la pertinencia de efectuar un análisis factorial exploratorio (AFE), tras el cual se llevó a cabo un análisis factorial confirmatorio (AFC) mediante el programa SPSS-AMOS (versión 22), implementándose el método de máxima verosimilitud, bajo el supuesto de una distribución normal multivariada y los indicadores de métodos robustos. Se utilizó una combinación de los siguientes índices para el ajuste absoluto: (a) Chi-cuadrado $\left(\chi^{2}\right.$, significación: $\left.\geq .05\right)$ y (b) razón de Chi-cuadrado/grados de 
libertad $\left(\chi^{2} / g l,<3.00\right)$. Para el ajuste comparativo se realizó: (a) Índice de bondad de ajuste comparativo (CFI, $\geq .90$ ), (b) índice de Tucker-Lewis (TLI, $\geq .90$ ) y otros índices: (a) Índice de Ajuste Normalizado (NFI, $\geq .90$ ); (b) Índice de Ajuste Relativo (RFI, $\geq .90$ ), (c) Índice de Ajuste Incremental (IFI, $\geq .90)$ y (d) raíz de residuo cuadrático promedio de aproximación (RMSEA, < .08 [IC90\%]) (Boomsma, 2000; MCDonald \& Moon-Ho, 2002).

Para examinar la consistencia interna de la escala y sus subescalas se utilizó la prueba alfa de Cronbach, aceptándose un índice mínimo de .60 , mientras que, para examinar la validez de constructo del instrumento, se implementaron análisis de correlación de Pearson, entre los puntajes obtenidos en las subescalas de la CDAQ (Borrajo, Gámez-Guadix, Pereda et al., 2015) y las escalas de la CEPA (Rey et al., 2019). Se consideraron débiles las correlaciones entre -.3 y -.1 y entre .1 y .3 , moderadas las correlaciones entre -.5 y -.3 y entre .3 y .5 y fuertes las que oscilaban entre -1 y -.5 y entre 1 y .5 .

\section{Resultados}

En la escala de victimización, la prueba KMO arrojó un índice de .914, mientras que la prueba de esfericidad de Bartlett evidenció resultados estadísticamente significativos: $X[8997.956]=171, p=.000$. En la escala de perpetración, la prueba KMO arrojó un valor de .887 , con resultados también estadísticamente significativos en la prueba de esfericidad de Bartlett: $X[8873.941]=210, p=.000$. Estos resultados indicaron la conveniencia de realizar el AFE, y se encontró que todos los ítems presentaban cargas factoriales superiores a .30 y se agruparon en dos factores, tanto en la escala de perpetración como en la de victimización, de manera similar a la validación de la versión original del instrumento. Los ítems que conformaron el primer factor de la escala de perpetración explicaron el $19.59 \%$ de la varianza total y, tal como lo plantean las autoras del instrumento, se refieren a conductas dirigidas a vigilar a la pareja o expareja e invadir su privacidad revisando sus conexiones en línea y utilizando sus claves sin su consentimiento, por lo que las agruparon en la subescala de "monitoreo/control" (Borrajo, Gámez-Guadix, Pereda et al., 2015).

Los ítems del segundo factor explicaron el $19.08 \%$ de la varianza total y se refieren a conductas que buscan generar dolor deliberadamente en la pareja o expareja: amenazas, insultos, la propagación de información privada y la suplantación de su identidad. Las anteriores conforman la subescala de "agresiones directas" (Borrajo, Gámez-Guadix, Pereda et al., 2015). El alfa obtenido por la escala de perpetración fue .84 , mientras que la subescala de monitoreo/ control alcanzó un alfa de .75 y la subescala de agresiones directas un alfa de .79 .

En la escala de victimización, el AFE evidenció dos factores que se ajustaban y agrupaban de la misma forma que en la escala de perpetración. El primer factor (monitoreo/ control) explicó el $19.31 \%$ de la varianza, mientras que el segundo factor (agresiones directas) explicó el 15.20\% de la varianza. La escala de victimización obtuvo un alfa de .87, con un alfa de .82 para la subescala monitoreo/control y de .80 , para la de agresiones directas.

Esta estructura de cuatro factores fue sometida a prueba con el AFC, asumiendo que estos factores estaban rela-

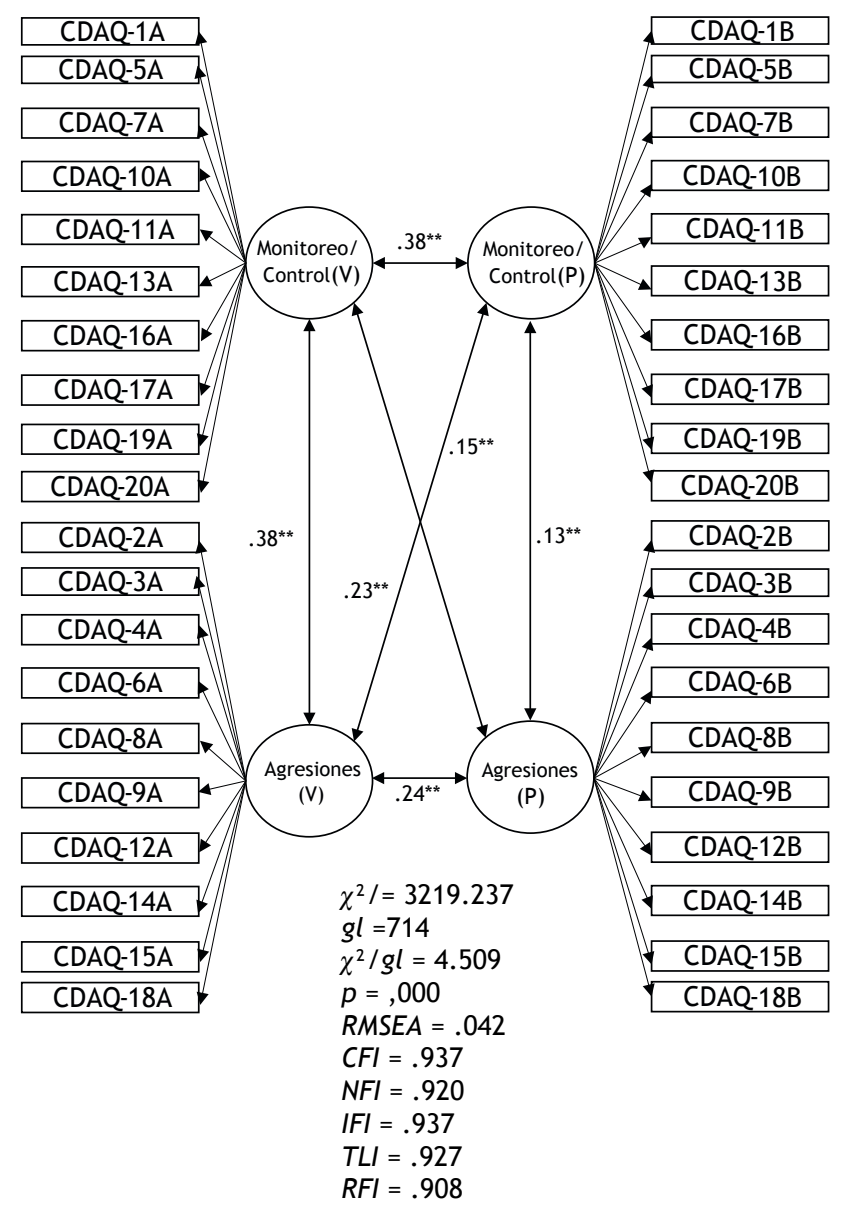

Figura 1. Análisis factorial confirmatorio de la CDAQ (Borrajo, Gámez-Guadix, Pereda et al., 2015). V: Victimización. P: Perpetración.

${ }^{* *} p \leq .01$

cionados entre sí y que cada pareja de ítems compartía los errores. Los índices obtenidos reflejan un ajuste aceptable (véase la figura 1). Sin embargo, se calculó la independencia entre la distribución de las conductas de CVN entre el grupo de adolescentes y adultos jóvenes y se encontró que los adolescentes informaron un promedio significativamente mayor de conductas agresivas ejercidas y sufridas y conductas de monitoreo/control perpetradas (véase la tabla 1), por lo que se procedió hacer un análisis de la invarianza del modelo de medida en dos diferentes escenarios. En relación con los adolescentes, los índices de bondad de ajuste obtenidos mediante el AFC señalan un buen ajuste al modelo de cuatro factores: $\chi^{2}=2393.791(p=.000) ; \chi^{2}$ $\mathrm{gl}=3.353 ; \mathrm{CFI}=.940 ; \mathrm{TLI}=.931 ; \mathrm{NFI}=.917 ; \mathrm{RFI}=.905 ;$ $I F I=.940$ y RMSEA = .039 (IC90\%: .038-.041). Sin embargo, entre los adultos jóvenes, estos índices fueron inferiores a los valores considerados como aceptables, según Boomsma (2000) y McDonald y Ho (2002): $\chi^{2}=3310.328(p=.000)$; $\chi^{2} / g l=4.636 ; C F I=.878 ; T L I=.845 ; N F I=.835 ; R F I=.895 ;$ $I F I=.880$ y $R M S E A=.084$ (IC90\%: .081-.087).

Entre los adolescentes, el alfa obtenido por la escala de perpetración fue .82, mientras que la subescala de monitoreo/control alcanzó un alfa de .72 y la de agresiones directas un alfa de .76, en tanto que entre los adultos jóvenes se evidenciaron valores alfa de $.83, .82$ y .76, 
Tabla 1 Diferencias entre adolescentes $(n=1511)$ y adultos jóvenes $(n=512)$ en la frecuencia de conductas de CVN

\begin{tabular}{|c|c|c|c|c|c|c|c|}
\hline & Factor & Grupo & Media & DS & $t$ & $p$ & $d$ de Cohen \\
\hline \multirow{4}{*}{ Victimización } & \multirow{2}{*}{ Agresiones } & Adolescentes & 18.87 & 7.53 & \multirow{2}{*}{14.405} & \multirow{2}{*}{.000} & \multirow{2}{*}{.74} \\
\hline & & Adultos & 13.77 & 4.66 & & & \\
\hline & \multirow{2}{*}{ Monitoreo/Control } & Adolescentes & 18.60 & 7.52 & \multirow{2}{*}{1.455} & \multirow{2}{*}{.146} & \multirow{2}{*}{.07} \\
\hline & & Adultos & 18.01 & 9.11 & & & \\
\hline \multirow{4}{*}{ Perpetración } & \multirow{2}{*}{ Agresiones } & Adolescentes & 18.43 & 7.38 & \multirow{2}{*}{17.817} & \multirow{2}{*}{.000} & \multirow{2}{*}{.91} \\
\hline & & Adultos & 12.47 & 2.88 & & & \\
\hline & \multirow{2}{*}{ Monitoreo/Control } & Adolescentes & 17.24 & 6.33 & \multirow{2}{*}{6.464} & \multirow{2}{*}{.000} & \multirow{2}{*}{.33} \\
\hline & & Adultos & 15.16 & 6.17 & & & \\
\hline
\end{tabular}

Nota. DS: Desviación estándar; $t$ : Valor de la prueba T de Student, $p$ : Probabilidad; $d$ de Cohen: Medida del tamaño del efecto. ${ }^{* *} p \leq .001$

respectivamente. En cuanto a la escala de victimización, entre los adolescentes alcanzó un alfa de .85, la subescala de monitoreo/control un alfa de .78 y la de agresiones directas un alfa de .78, mientras que, entre los adultos jóvenes, se obtuvieron valores alfa de .89, .89 у .79, respectivamente.

En la tabla 2 se presenta la matriz de correlaciones obtenidas. Como se puede observar, las dos subescalas (agresiones directas y monitoreo/control) correlacionaron fuertemente entre sí, tanto en la escala de perpetración como en la de victimización, y fueron moderadas las correlaciones entre las dos subescalas cruzadas por escala, es decir, entre las puntuaciones de la subescala de agresiones directas de perpetración y las de la subescala de control/ monitoreo de victimización y viceversa. Las correlaciones con las escalas de la CEPA (Rey et al., 2019), asimismo, fueron estadísticamente significativas, particularmente con la escala de control de dicho instrumento, con la cual las correlaciones fueron moderadas.

\section{Discusión}

El objetivo de este estudio fue evaluar la validez y la confiabilidad de la CDAQ (Borrajo, Gámez-Guadix, Pereda et al., 2015) con una muestra de adolescentes y adultos jóvenes colombianos entre 13 y 40 años. El AFE evidenció que los 20 ítems del instrumento se agrupaban en cuatro factores consistentes con las cuatro subescalas de la versión original del instrumento ("agresiones directas" y "monitoreo/ control" de perpetración y de victimización), estructura que fue ratificada con índices de ajuste satisfactorios a través del $A F C$. Los índices alfa obtenidos por las dos escalas y las cuatro subescalas también fueron satisfactorios. A diferencia de lo informado por los autores de la versión original del instrumento, en este estudio las subescalas de monitoreo/control explicaron un porcentaje un poco mayor de la varianza que las subescalas de agresiones directas, lo que sugiere que en la muestra colombiana eran más comunes las conductas del primer tipo que las segundas, similar a lo reportado por Lara (2020) con estudiantes chilenos.

No obstante, cuando se examinaron los índices de ajuste por grupo de edades, estos indicaron un buen ajuste para los adolescentes, pero no para los adultos jóvenes, lo cual sugiere que el instrumento es más adecuado para examinar la CVN entre los adolescentes, aunque se recomiendan otros estudios comparativos realizados con este cuestionario. Se encontró también que la CVN era más frecuentemente ejercida y sufrida por los adolescentes que por los adultos jóvenes, lo cual es coherente con los resultados del estudio de Villa et al. (2017) y podría explicarse por aspectos como la frecuencia de uso de medios digitales, la experiencia en relaciones románticas y la presión de los pares, entre otros factores que se deberían examinar en el futuro.

En relación con la matriz de correlaciones obtenidas, se destaca el hecho de que las dos subescalas (agresiones directas y monitoreo/control) correlacionaron fuertemente entre sí, tanto en la escala de perpetración como en la de victimización, lo que indica que el instrumento mide el mismo constructo. A su vez, las correlaciones fueron moderadas, pero no fuertes entre las dos subescalas cruzadas por escala, es decir, entre las puntuaciones de la

Tabla 2 Correlación entre los factores que conforman la CDAQ (Borrajo, Gámez-Guadix, Pereda et al., 2015) y la Lista de Chequeo de Experiencias de Maltrato Psicológico en la Pareja (Rey et al., 2019)

\begin{tabular}{|c|c|c|c|c|c|c|c|c|}
\hline & & \multicolumn{2}{|c|}{ Victimización online } & \multicolumn{2}{|c|}{ Perpetración online } & \multicolumn{3}{|c|}{ Violencia psicológica offline } \\
\hline & & Agresiones & Control & Agresiones & Control & Coerción & Humillación & Control \\
\hline \multirow{2}{*}{$\begin{array}{l}\text { Victimización } \\
\text { online }\end{array}$} & Agresiones & 1 & & & & & & \\
\hline & Control & $.620^{* *}$ & 1 & & & & & \\
\hline \multirow{2}{*}{$\begin{array}{l}\text { Perpetración } \\
\text { online }\end{array}$} & Agresiones & $.656^{* *}$ & $.309^{* *}$ & 1 & & & & \\
\hline & Control & $.474^{* *}$ & $.596^{* *}$ & $.628^{* *}$ & 1 & & & \\
\hline \multirow{3}{*}{$\begin{array}{l}\text { Violencia } \\
\text { psicológica } \\
\text { offline }\end{array}$} & Coerción & $.244^{* *}$ & $.144^{* *}$ & $.203^{* *}$ & $.152^{* *}$ & 1 & & \\
\hline & Humillación & $.349^{* *}$ & $.271^{* *}$ & $.302^{* *}$ & $.278^{* *}$ & $.405^{* *}$ & 1 & \\
\hline & Control & $.270^{\star *}$ & $.308^{* *}$ & $.316^{* *}$ & $.403^{* *}$ & $.236^{* *}$ & $.448^{* *}$ & 1 \\
\hline
\end{tabular}

${ }^{* *} p \leq .01$ 
subescala de agresiones directas de perpetración y las de la subescala de control/monitoreo de victimización y viceversa, lo que sugiere que el instrumento mide dos elementos diferentes de la CVN.

Por otra parte, las correlaciones con las escalas de la CEPA (Rey et al., 2019) fueron estadísticamente significativas, particularmente con la escala de control de dicho instrumento, lo que constituye una evidencia de la validez de constructo del instrumento, si se tiene en cuenta que las conductas de control, coerción y agresiones realizadas por medios electrónicos constituyen una forma de violencia, principalmente psicológica, manifestada a través de estos medios (Temple et al., 2016). En la validación de la versión original del instrumento, se obtuvieron correlaciones estadísticamente significativas con la VN offline de tipo psicológico, las cuales también fueron moderadas con las subescalas de monitoreo/control del instrumento (Borrajo, Gámez-Guadix, Pereda et al., 2015). Lara (2020) también reportó correlaciones moderadas o altas entre las escalas de este instrumento y varios tipos de VN psicológica. Caridade et al. (2020), en la misma línea, informaron cifras elevadas de CVN y VN offline de tipo psicológico.

Así, estos resultados, junto a los anteriores, señalan que este instrumento tiene adecuadas propiedades psicométricas $\mathrm{y}$, por lo tanto, podría arrojar resultados válidos y confiables si se aplica adecuadamente a adolescentes y adultos jóvenes colombianos con diferentes propósitos, por ejemplo, para detectar casos de CVN en instituciones educativas y otras poblaciones, examinar la prevalencia, las dificultades asociadas y los factores de riesgo de la CVN, y evaluar estrategias de prevención e intervención.

Como fortalezas de esta investigación se pueden mencionar el tamaño de la muestra, la participación de mujeres y varones de un rango mayor de edades que el que participó en el estudio original. Ello permitió examinar diferencias entre adolescentes y adultos jóvenes y el uso de un instrumento previamente validado para examinar la validez de constructo del instrumento. No obstante, como todos los instrumentos de autoinforme, es posible que las fallas de memoria normales y la deseabilidad social hayan influido en las respuestas dadas. Además, no se examinaron otras variables que se han relacionado con la CVN, para obtener más evidencias de su validez convergente, como la VN física y sexual y el cyberbullying (Borrajo, Gámez-Guadix, Pereda et al., 2015; Hinduja \& Patchin, 2020; Peña et al., 2018; Stonard et al., 2014), aspectos que se podrían considerar en otros estudios sobre la validez del instrumento. De igual manera, se recomienda examinar la validez y la confiabilidad del instrumento con adolescentes y jóvenes de otras regiones del país.

\section{Financiación}

Este artículo es producto de un proyecto de investigación aprobado y apoyado financieramente por la Dirección de Investigaciones de la Universidad Pedagógica y Tecnológica de Colombia. Código: SGI-2654.

\section{Referencias}

Alegría, M., \& Rodríguez, A. (2015). Violencia en el noviazgo: Perpetración, victimización y violencia mutua. Una revisión. Actualidades en Psicología, 29(118), 57-72. https://doi. org/10.15517/ap.v29i118.16008

Asociación de la Industria Móvil de Colombia. (2017). Hábitos de uso de telecomunicaciones móviles en Colombia. https:// bit.ly/3xfZbHM

Borrajo, E., \& Gámez-Guadix, M. (2015). Comportamientos, motivos y reacciones asociadas a la victimización del abuso online en el noviazgo: un análisis cualitativo. Revista de Victimología, 2, 73-95. https://doi.org/10.12827/RVJV.2.04

Borrajo, E., \& Gámez-Guadix, M. (2016). Abuso online en el noviazgo: relación con depresión, ansiedad y ajuste diádico. Psicología Conductual, 24(2), 221-235.

Borrajo, E., Gámez-Guadix, M., \& Calvete, E. (2015). Cyber dating abuse: Prevalence, context, and relationship with offline dating aggression. Psychological Reports, 116(2), 565-585. https://doi.org/10.2466/21.16.PR0.116k22w4

Borrajo, E., Gámez-Guadix, M., Pereda, N., \& Calvete, E. (2015). The development and validation of the cyber dating abuse questionnaire among young couples. Computers in Human Behavior, 48, 358-365. https://doi.org/10.1016/j.chb.2015.01.063

Boomsma, A. (2000). Reporting analyses of covariance structures. Structural Equation Modeling: A Multidisciplinary Journal, 7(3), 461-483. https://doi.org/10.1207/S15328007SEM0703_6

Caridade, S., Ataíde., I., \& Dinis, A. (2020). Cyber dating abuse victimization and association with offline dating violence. En M. L. Knudsen (Ed.), Victims of Violence: Support, Challenges and Outcomes (pp. 231-250). Nova Science Publishers.

Caridade, S., Braga, T., \& Borrajo, E. (2019). Cyber dating abuse (CDA): Evidence from a systematic review. Aggression \& Violent Behavior, 48, 152-168. https://doi.org/10.1016/j.avb.2019.08.018

Cava, M. J., \& Buelga, S. (2018). Psychometric properties of the Cyber-Violence Scale in Adolescent Couples (Cib-VPA). Suma Psicológica, 25(1), 51-61. https://doi.org/10.14349/sumapsi.2018. v25.n1.6

Centers for Disease Control and Prevention. (2019). Teen dating violence. https://www.cdc.gov/features/DatingViolence/

Dardis, C., Edwards, K., Kelley, E., \& Gidycz, C. (2017). Perceptions of dating violence and associated correlates: A study of college young adults. Journal of Interpersonal Violence, 32(21), 32453271. https://doi.org/10.1177/0886260515597439

Deloitte (2019). Consumo móvil en Colombia. https://bit. ly/2UnHcAD

Gámez-Guadix, M., Borrajo, E., \& Calvete, E. (2018). Abuso, control y violencia en la pareja a través de internet y los smartphones: características, evaluación y prevención. Papeles del Psicólogo, 39(3), 218-227. https://doi.org/10.23923/pap. psicol2018.2874

Gracia-Leiva, M., Puente-Martínez, A., Ubillos-Landa, S., \& PáezRovira, D. (2019). Dating violence (DV): A systematic metaanalysis review. Annals of Psychology, 35(2), 300-313. https:// doi.org/10.6018/analesps.35.2.333101

Hinduja, S., \& Patchin, J. (2020). Digital dating abuse among a national sample of U.S. youth. Journal of Interpersonal Violence. https://doi.org/10.1177/0886260519897344

Hoefer, R., Black, B., \& Ricard, M. (2015). The impact of state policy on teen dating violence prevalence. Journal of Adolescence, 44, 88-96. https://doi.org/10.1016/j.adolescence.2015.07.006 
Lara, L. (2020). Cyber dating abuse: Assessment, prevalence, and relationship with offline violence in young Chileans. Journal of Social and Personal Relationships, 37(5), 1681-1699. https:// doi.org/10.1177/0265407520907159

Luengo, J. (2014). Ciberbullying: prevenir y actuar. Colegio Oficial de Psicólogos de Madrid.

Martínez, J. A., Vargas, R., \& Novoa, M. (2016). Relación entre la violencia en el noviazgo y observación de modelos parentales de maltrato. Psychologia: Avances de la Disciplina, 10(1), 101-112.

Martínez-Gómez, J., Bolívar-Suárez, Y., Rey-Anacona, C., Ramírez-Ortiz, L., Lizarazo-Ojeda, A., \& Yanez-Peñúñuri, L. (2021). Esquemas tradicionales de roles sexuales de género, poder en las relaciones y violencia en el noviazgo. Revista Iberoamericana de Psicología y Salud, 12(1), 107-121. https://doi. org/10.23923/j.rips.2021.01.041

McDonald, R., \& Moon-Ho, R. (2002). Principles and practice in reporting structural equation analyses. Psychological Methods, 7, 64-82. https://doi.org/10.1037/1082-989X.7.1.64

Montero, I., \& León, O. (2007). A guide for naming research studies in Psychology. International Journal of Clinical and Health Psychology, 7(3), 847-862.

Peña, F., Rojas-Solís, J., \& García-Sánchez, P. (2018). Uso problemático de internet, cyberbullying y ciberviolencia de pareja en jóvenes universitarios. Diversitas: Perspectivas en Psicología, 14(2), 205-219. https://doi.org/10.15332/ s1794-9998.2018.0014.01

Redondo, J., Inglés, C., \& García, K. (2017a). Papel que juega la edad en la violencia en el noviazgo de estudiantes de la Universidad Pontificia Bolivariana de Bucaramanga. Diversitas: Perspectivas en Psicología, 13(1), 41-54. https://doi.org/10.15332/ s1794-9998.2017.0001.03

Redondo, J., Luzardo, M., García, K., \& Inglés, C. (2017b). Malos tratos durante el noviazgo en jóvenes universitarios: diferencias de sexo. I+ D Revista de Investigaciones, 9(1), 59-69.

Redondo, J., Luzardo-Briceño, M., García-Lizarazo, K., \& Inglés, C. (2017c). Impacto psicológico del cyberbullying en estudiantes universitarios: Un estudio exploratorio. Revista Colombiana de Ciencias Sociales, 8(2), 458-478. https://doi. org/10.21501/22161201.2061

Reed, L., Tolman, R., \& Ward, L. (2017). Gender matters: Experiences and consequences of digital dating abuse victimization in adolescent dating relationships. Journal of Adolescence, 59, 79-89. https://doi.org/10.1016/j.adolescence.2017.05.015

Rey, C. A. (2009). Maltrato en el noviazgo de tipo físico, psicológico, emocional, sexual y económico: Un estudio exploratorio. Acta Colombiana de Psicología, 12(2), 27-36.

Rey-Anacona, C. (2013). Prevalencia y tipos de maltrato en el noviazgo en adolescentes y adultos jóvenes. Terapia Psicológica, 31(2), 143-154. https://doi.org/10.4067/ S0718-48082013000200001

Rey, C., Martínez, J., Herrero Olaizola, J., \& Rodríguez, F. (2019). Psychometric properties of the Checklist of Experiences of Psychological Abuse to the Couple in adolescents and young adults. Journal of Interpersonal Violence. https://doi. org/10.1177/0886260518823294

Rubio-Garay, F., López-González, M., Carrasco, M., \& Amor, P. (2017). Prevalencia de la violencia en el noviazgo: Una revisión sistemática. Papeles del Psicólogo, 38(2), 135-147. https://doi. org/10.23923/pap.psicol2017.2831

Smith, K., Cénat, J., Lapierre, A., Dion, J., Hébert, M., \& Côté, K. (2018). Cyber dating violence: Prevalence and correlates among high school students from small urban areas in Quebec. Journal of Affective Disorders, 234, 220-223. https://doi. org/10.1016/j.jad.2018.02.043

Stonard, K., Bowen, E., Lawrence, T., \& Price, S. (2014). The relevance of technology to the nature, prevalence and impact of adolescent dating violence and abuse: A research synthesis. Aggression and Violent Behavior, 19(4), 390-417. https://doi. org/10.1016/j.avb.2014.06.005

Temple, J., Choi, H., Brem, M., Wolford-Clevenger, C., Stuart, G., Peskin, M., \& Elmquist, J. (2016). The temporal association between traditional and cyber dating abuse among adolescents. Journal of Youth Adolescence, 45(2), 340-349. https://doi. org/10.1007/s10964-015-0380-3

Villa, M., García, A., Cuetos. G., \& Sirvent. C. (2017). Violencia en el noviazgo, dependencia emocional y autoestima en adolescentes y jóvenes españoles. Revista Iberoamericana de Psicología y Salud, 8(2), 96-107. https://doi.org/10.23923/j. rips.2017.08.009

Wincentak, K., Connolly, J., \& Card, N. (2017). Teen dating violence: A meta-analytic review of prevalence rates. Psychology of Violence, 7(2), 224-241. https://doi.org/10.1037/a0040194 